\title{
Obtenção de patentes na indústria do Estado de São Paulo: uma análise utilizando regressão logística
}

\author{
Antônio Carlos Pacagnella Júnior UFSCar \\ Geciane Silveira Porto FEARP/USP \\ Sérgio Kannebley Júnior FEARP/USP \\ Sérgio Luís da Silva UFSCar \\ Carlos Alberto Grespan Bonacim FEA-USP
}

\section{RESUMO}

Este artigo tem como proposta central analisar as variáveis de influência na obtenção de patentes da indústria paulista, utilizando, para isto, dados da Pesquisa de Atividade Econômica Paulista (PAEP), realizada pela Fundação Sistema Estadual de Análise de Dados (SEADE), considerando o período de 1999 a 2001. Trata-se de uma pesquisa com abordagem quantitativa, de caráter descritivo e explicativo, na qual foi utilizada a técnica de regressão logística. Os resultados encontrados mostram a orientação exportadora, a origem do capital controlador, a origem principal de receita (bens ou serviços), o fator relacionado aos investimentos em pesquisa e desenvolvimento (P\&D), a presença de laboratório ou departamento de P\&D, a cooperação em P\&D e as fontes de informação para atividades inovativas são variáveis significativas de influência na probabilidade de obtenção de patentes por parte das empresas industriais paulistas.

\section{Patent obtaining in the São Paulo State industry: an analysis using logistic regression}

\begin{abstract}
This paper aims at analyzing the influence variables of patent obtaining by the industries from São Paulo State, using to this, data from Pesquisa de Atividade Econômica Paulista (PAEP) provided by Fundação Sistema de Análise de Dados (SEADE) from 1999 to 2001. The research has a quantitative approach with descriptive and correlational characteristics, where the statistical method used was the logistic regression. The results show that the export orientation, the capital origin, the main outcome source (goods or services), the factor related to the investments on research and development, (R\&D) availability of $R \& D$ specific department or laboratory, $R \& D$ cooperation and the information sources for innovative activities are significant influence variables on the probability of patent obtaining.
\end{abstract}

\section{KEY WORDS}

Patents, technological innovation, logistic regression. 


\section{INTRODUÇÃO}

As patentes são instrumentos utilizados pelas organizações para proteger suas invenções e inovações, constituindo uma alternativa ao segredo industrial, ao "know-how", aos acordos de licenciamento e outras maneiras que buscam garantir a exclusividade da exploração comercial da tecnologia desenvolvida. Trata-se de um artifício legal, utilizado para impedir a apropriação da propriedade intelectual por terceiros, que visam a sua utilização para benefício próprio.

\section{Aque as organizações protejam cada vez}

\section{mais seu capital intelectual.}

Uma patente é um título de propriedade temporário, emitido no Brasil pelo Instituto Nacional de Propriedade Intelectual (INPI) e internacionalmente por órgãos como o United States Patent Office (USPTO) e European Patent Office (EPO), que permite ao seu proprietário fazer uso do objeto da patente de forma exclusiva durante determinado período de tempo.

A patenteabilidade de uma determinada tecnologia depende, de acordo com Humphreys (2006), de três fatores: novidade, quesito que define se o objeto reivindicado não existe e nem está acessível, atividade inventiva, que define que o objeto da patente deve estar no "estado da técnica", ou seja, que para um técnico do assunto o objeto não decorra de forma óbvia e evidente, e a aplicação industrial, devendo o objeto ser aplicável em um sentido amplo, não necessariamente de forma imediata ou economicamente viável.

Por estarem diretamente associadas ao desenvolvimento de tecnologias, a análise das patentes tem sido utilizada em diversos estudos como indicador de inovação tecnológica, permitindo verificar o desempenho tecnológico de empresas, regiões, setores industriais e mesmo de países. Corroborando estas afirmações, Abraham e Moitra (2001) argumentam que os estudos sobre patentes utilizam-nas como uma ferramenta para indicar o desenvolvimento tecnológico e o crescimento econômico no contexto nacional e internacional.

Ainda segundo Abraham e Moitra (2001), no ambiente empresarial a análise de patentes também é de grande importância, pois serve como base para a avaliação das políticas sobre P\&D, estimação das forças e fraquezas dos concorrentes e da identificação de oportunidades de exploração de novos mercados.
A escolha pelo Estado de São Paulo se justifica por se tratar do estado mais industrializado e de maior importância econômica para o Brasil, além disso, de acordo com a Fapesp (2004), as empresas industriais paulistas respondem por aproximadamente $56 \%$ do dispêndio privado em P\&D no Brasil e por cerca de $51 \%$ das patentes depositadas no Instituto Nacional de Propriedade Industrial.

A Figura 1, extraída de Albuquerque et alii (2002), apresenta a distribuição espacial no mapa brasileiro das patentes obtidas em cada município, verificando-se uma grande concentração no Estado de São Paulo quando se compara com o resto do país. Os autores ainda destacam que o Estado é responsável por cerca de $32 \%$ do total de pesquisadores e $47 \%$ do total de artigos científicos publicados no país, reforçando a relevância de sua contribuição científica e tecnológica.

A partir dos argumentos supracitados, da importância das patentes como indicadores de inovação tecnológica e de crescimento econômico e da indústria do Estado de São Paulo também quanto a estes dois quesitos, o presente trabalho tem por objetivo analisar as variáveis de influência na obtenção de patentes por parte das empresas industriais paulistas, utilizando-se para este fim a técnica de regressão logística.

Espera-se que este trabalho possa contribuir para o entendimento dos mecanismos associados à obtenção de patentes por parte da indústria paulista, de forma a fornecer subsídios para uma compreensão mais profunda da sua dinâmica inovativa e para o desenvolvimento de leis e políticas públicas que incentivem o desenvolvimento tecnológico, melhorando a competitividade destas organizações.

\section{PATENTES}

A economia baseada no conhecimento exige que as organizações protejam cada vez mais seu capital intelectual, de forma a manter competências essenciais que subsidiem sua competitividade (CHOY; YEW; LIN, 2006).

Com o auxílio da utilização de patentes, é possível assegurar vantagem competitiva por meio da exploração comercial exclusiva de um determinado tipo de conhecimento com proteção legal, desencorajando eventuais cópias ou plágio por outras organizações.

Além disso, segundo Hou e Lin (2006), a posse de patentes permite ao seu dono outros benefícios em negócios relacionados às mesmas, como a venda de produtos patenteados ou o licenciamento para a exploração da patente. Os autores ainda afirmam que também existem benefícios para quem 
adquire as patentes, como menores investimentos $\mathrm{P} \& \mathrm{D}$ e a redução do risco inerente ao desenvolvimento de novas tecnologias.

Embora os motivos que levam a organização a patentear tecnologias possam variar segundo diversos aspectos, como o tamanho ou o mercado onde ela atua, Olsson e McQueen (2000) argumentam que de forma geral existem nove razões para que se busque a proteção da propriedade intelectual por meio de patentes:

(1) As características comerciais das invenções da organização (potencial de comércio);

(2) Aspectos financeiros (obtenção de financiamento ou capital de risco);

(3) Deserção de pessoal;

(4) Oportunidades de licenciamento;

(5) Desejo de bloquear competidores;

(6) Reconhecimento do inventor;

(7) Melhorar a imagem do produto da empresa;

(8) Baixa efetividade de segredo industrial;

(9) Baixa efetividade de outras formas de manter a exclusividade da invenção.

No Brasil existem três modelos de patentes: o primeiro é o Privilégio de Invenção (PI), aplicado para inventos inéditos e com prazo de proteção de vinte anos; o segundo é o Modelo de Utilidade (MU), aplicado a inventos e soluções técnicas novas, que consistem de melhora na função, uso ou aplicação do objeto e que possui prazo de proteção de quinze anos; o terceiro é o Desenho Industrial (DI), aplicado para proteger durante dez anos a forma ou o formato construtivo de objeto.

O processo brasileiro de registro de patentes é composto, segundo Humphreys (2006), por quatro fases: Depósito, quando o requerente apresenta um relatório descritivo do objeto patenteado, desenhos e outros; Publicação, declaração pública do objeto de patente (o que muitas vezes leva a organização a preferir o segredo industrial à patente); Exame, fase em que se avalia a patenteabilidade do objeto; e Decisão, que em caso favorável garante a "carta-patente" ao solicitante.

Além de se tratar de um importante instrumento de proteção intelectual, as patentes são de suma relevância para o processo de difusão tecnológica, segundo Encaoua, Guellec e Martinez (2006). Isto acontece devido ao fato de que para se obter uma patente, e consequentemente a exclusividade de exploração de seu conteúdo, o inventor deve divulgá-lo, o que pode dar origem a novas tecnologias.

Este último ponto é reforçado por Burke e Reitzig (2007), que apontam para a importância das patentes enquanto elemento estimulante para a pesquisa e o desenvolvimento e para facilitar a transferência de tecnologias.
Outro aspecto importante sobre as patentes é a sua utilização como indicadores da capacidade tecnológica das organizações. A análise do número de patentes obtidas, embora seja uma medida útil, gera dois problemas significativos, segundo Coombs e Bierly (2006): o primeiro é o fato de existirem tecnologias não patenteáveis e o segundo está relacionado ao princípio de que patentes diferem enormemente umas das outras de acordo com aspectos técnicos e econômicos.

O primeiro problema está relacionado à incapacidade de proteção de uma patente sobre determinados tipos de conhecimento, como fórmulas ou algoritmos matemáticos (incluindo sua aplicação em softwares), teorias científicas, matérias biológicas e outros. Já o segundo problema reflete o fato de que patentes são diferentes em termos de valor, que pode ser avaliado, segundo Reitzig (2001), por meio dos seguintes aspectos:

- Tempo de vida: o tempo de validade de uma patente determina seu valor; quanto maior este período, maior a possibilidade de que ela traga retorno financeiro;

- Grau de novidade: distância entre a tecnologia atual e a da invenção patenteada;

- Usos ou funções das patentes: as possibilidades de utilização do objeto patenteado podem aumentar seu valor;

- Dificuldade de invenção: está relacionada com a dificuldade imposta pela obtenção da patente no que se refere à invenção de algo semelhante pelos concorrentes;

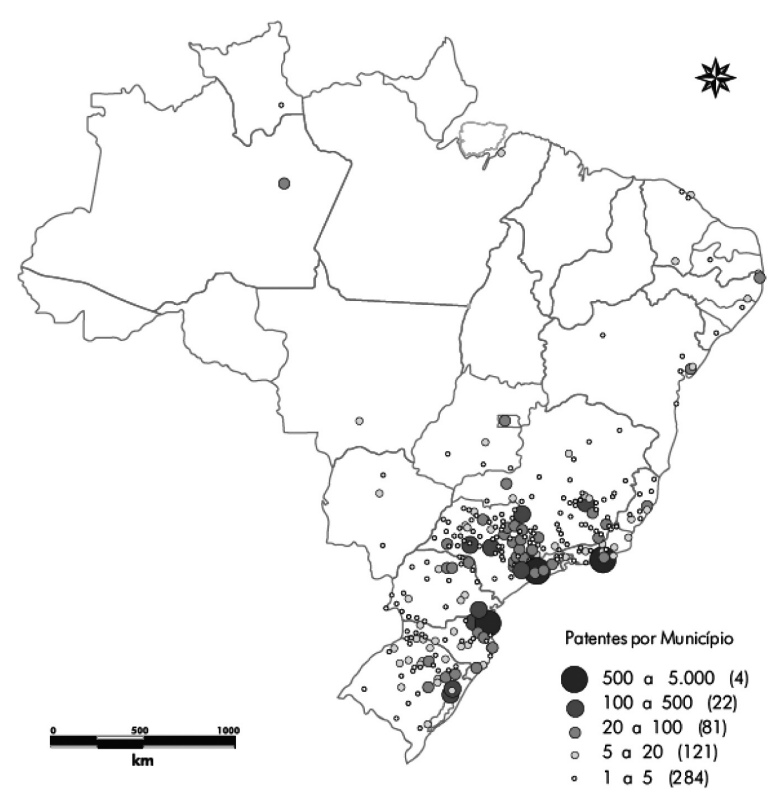

Figura 1: Patentes por município.

Fonte: Albuquerque et alii (2002) 
- Posição no portfólio: a importância da patente vai variar dependendo do portfólio de patentes do mesmo tipo que existirem;

- Abrangência da patente: patentes podem diferir quanto ao grau de proteção oferecido para a invenção; assim, quanto mais abrangente melhor;

- Capacidade de barganha: está relacionada com a capacidade comercial da patente e de como ela pode ser utilizada como moeda de troca entre empresas, ou mesmo como produto;

- Exclusão de direitos: relacionada à vantagem que a patente oferece à empresa que a obtém ao conseguir bloquear concorrentes no desenvolvimento de tecnologias;

- Abertura ("disclosure"): a abertura de informações técnicas pela patente pode fazer seu valor aumentar, já que permite que outras empresas analisem a possibilidade de sua utilização, o que aumenta sua capacidade de barganha.

Fora do contexto da capacidade tecnológica das organizações, ressalta-se que, no que tange ao seu uso como indicador, as patentes têm sido amplamente utilizadas em análises sobre inovação tecnológica em setores, regiões e mesmo para comparação internacional entre países, destacando-se como exemplo a publicação Compendium of Patent Statistics, publicada anualmente pela Organisation for Economic Co-operation and Development (OCDE).

\section{utro aspecto importante sobre as patentes é a sua utilização como indicadores da capacidade tecnológica das organizações.}

Sobre este aspecto, vale destacar que embora o Estado de São Paulo se destaque no âmbito nacional como citado anteriormente, a participação do Brasil no âmbito internacional é pequena; para se ter uma idéia, as patentes brasileiras registradas no USPTO representam menos de $0,005 \%$ do total.

Entretanto, de acordo com a OCDE (2007), o número de patentes de países emergentes como o Brasil tem crescido rapidamente, aumentando a participação em mais de cinco vezes no período de 1991 até 2002; por isso, estudos que permitam avançar a discussão sobre o assunto no Brasil são de fundamental importância para promover melhores políticas públicas de incentivo à geração de novas tecnologias, sua adequada proteção e consequente difusão (como já discutido previamente), pelo uso de patentes.

\section{ASPECTOS METODOLÓGICOS}

No meio acadêmico é comum classificar as pesquisas em relação a seus objetivos gerais, o que é de grande utilidade para estabelecer uma aproximação conceitual com o objeto de estudo.

Desta forma, sob o ponto de vista dos objetivos, este trabalho apresenta elementos de pesquisas descritivas, que visam primordialmente a descrição das características de determinadas populações ou fenômenos, como também a descrição de um processo numa organização, o estudo do nível de atendimento de entidades, levantamento de opiniões e atitudes, além disso, também são pesquisas descritivas aquelas que visam descobrir a existência de associações entre variáveis (GIL, 2002).

Ainda quanto aos objetivos, Dane (1990) afirma que uma pesquisa pode apresentar mais de um tipo. Assim, o presente trabalho também possui elementos de pesquisas explicativas, que têm como preocupação central a identificação dos fatores que determinam ou que contribuem para a ocorrência dos fenômenos, suas causas e consequências (RICHARDSON, 1999).

A abordagem utilizada neste estudo é a quantitativa e utiliza dados secundários provindos da Pesquisa de Atividade Econômica Paulista (PAEP), realizada no ano de 2002 e que abrange o período que vai de 1999 a 2001. Para processo de amostragem, a Fundação SEADE utilizou o Cadastro de Empresas (CEMPRE), fornecido pelo Instituto Brasileiro de Geografia e Estatística (IBGE), onde foram selecionados 1.006.037 registros, dando origem a uma amostra de 42.023 empresas de 21 setores industriais (ou estratos) que seguem a Classificação Nacional de Atividades Econômicas (CNAE) de dois dígitos.

\section{DESCRIÇÃO DA AMOSTRA}

A Tabela 1 apresenta os estratos da amostra da PAEP, trazendo informações sobre a composição de cada setor em termos do total de empresas e o percentual de empresas que obtiveram patentes.

Os estratos da amostra (setores) seguiram a classificação do Cadastro Nacional de Atividades Econômicas (CNAE) de dois dígitos, devendo-se destacar que a quantidade de empresas presentes em cada estrato é diferente, sendo pequena em alguns casos, que foram agrupados na Indústria extrativa e em Outras indústrias, de forma a garantir sig- 
nificância estatística para todos os setores que compõem a amostra.

Desta forma é possível observar que os setores com maior participação na amostra são os setores de Produtos metálicos, com $11,98 \%$, Vestuário e acessórios, com $11,49 \%$, Outras indústrias, com $11,03 \%$, Alimentos e bebidas, com 9,92\%, e o setor de Máquinas e equipamentos, com $7,65 \%$ do total, enquanto que os setores com menor participação nesta composição são os setores de Refino de petróleo, com 0,19\%, Equipamentos de informática, com $0,30 \%$, Outros equipamentos de transporte, com 0,53\%, Equipamentos médicos, com 1,37\%, e a Indústria extrativa, com $1,38 \%$.

Quando se observam os dados referentes à obtenção de patentes, percebe-se em primeira instância o baixo número de empresas, sendo no total apenas 1.839 , ou $4,38 \%$ da amostra. Entre os setores é relevante destacar o setor de Máquinas e equipamentos, responsável pela maior parcela destas empresas $(19,14 \%)$, além do setor de Produtos químicos, que corresponde a apenas $4,70 \%$ do total da amostra, mas onde estão $12,45 \%$ das empresas que obtiveram patentes, e do setor de Borracha e Plásticos, que participa com $14,96 \%$ do total de empresas que obtiveram patentes no período considerado pela PAEP.

Também é possível citar como destaques "negativos" os setores de Refino de petróleo, onde nenhuma empresa obteve patentes, a Indústria extrativa, com apenas 0,27\% de empresas que obtiveram patentes, e o setor de Outros equipamentos de transporte, onde as empresas que patentearam representam apenas $0,44 \%$ do total.

Quanto à distribuição da amostra com relação à faixa de pessoal ocupado, é possível observar, pela Tabela 2, que a amostra da PAEP é composta em sua maior parcela por empresas de 5 a 29 pessoas, correspondendo a $78,27 \%$ do total. A segunda maior parcela é de empresas na faixa de 30 a 99 pessoas ocupadas, com $14,05 \%$ do total da amostra, enquanto que a faixa de 100 a 499 corresponde a $6,06 \%$ e a faixa de 500 ou mais pessoas ocupadas corresponde a apenas $1,62 \%$.

Para as empresas que obtiveram patentes, esta proporção se inverte, evidenciando a influência do tamanho. Assim, das empresas que estão na faixa de até 29 pessoas ocupadas, apenas $2,97 \%$ obtiveram patentes, enquanto que nas outras faixas este percentual aumenta sequencialmente com $6,88 \%$ na faixa de 30 a 99 pessoas ocupadas, $13,16 \%$ na faixa de 100 a 499 pessoas e $17,74 \%$ na faixa de 500 ou mais pessoas.

Outros aspectos relevantes sobre a amostra estão presentes na Tabela 3, que mostra a composição em termos de orientação exportadora e origem do capital controlador do total de empresas e das empresas que obtiveram patentes no período considerado pela PAEP.

A grande maioria das empresas que compõem a amostra, segundo os dados apresentados na Tabela 3, é de empresas não exportadoras, representando $83,82 \%$ do total, enquanto que as empresas exportadoras correspondem a 10,96\%. Entretanto, quando se observa a obtenção de patentes, verificase que entre as exportadoras o percentual de empresas que obtiveram patentes é de 15,07\% enquanto que entre as não exportadoras este percentual é de apenas 3,06\%, indicando uma possível influência da orientação exportadora na obtenção de patentes.

Da mesma forma, quando se trata da origem do capital controlador das empresas, observa-se que a imensa maioria é de empresas cujo capital controlador é de origem nacional, onde esta parcela é de $92,83 \%$ contra 2,66\% de empresas com capital controlador de origem internacional ou mista. Com respeito a obtenção de patentes, o percentual de empresas com capital controlador de origem internacional ou mista é de $21,22 \%$, bem superior ao percentual de empresas de capital controlador de origem nacional, que representam 3,99\% do total, indicando também uma possível influência deste fator na obtenção de patentes.

\section{TÉCNICA ESTATÍSTICA}

A regressão logística é uma técnica estatística que tem por objetivo discriminar dois grupos de observações dentro de uma amostra, diferindo da regressão linear por utilizar uma variável dependente dicotômica ou binária.

De acordo com Hosmer e Lemeshow (1989), a técnica de regressão logística tornou-se um método padrão para análise de regressão para variáveis binárias, sendo amplamente utilizada em situações da medicina (estado de saúde de um paciente), marketing (compra de produtos) e outras situações em que a análise de dados envolve prever o valor de uma variável de resultado categórico.

Trata-se, portanto, de uma técnica apropriada para este estudo, onde variável dependente é dicotômica, assumindo um entre dois resultados possíveis: "obteve ou não obteve patentes".

Para Hair (2005), esta técnica deriva seu nome da transformação logística usada com a variável dependente e que permite calcular diretamente a probabilidade do fenômeno analisado ocorrer.

Segundo Johnson e Wichern (1998), o modelo de regressão logística é baseado na função logística:

$$
f(z)=\frac{1}{1+e^{-(z)}},
$$


Podendo ser expresso na forma:

$$
z=\ln \left(\frac{p}{1+p}\right)=\alpha+\beta_{1} X_{1}+\beta_{2} X+\ldots+\beta_{3} X_{3}
$$

Onde:

$p=$ probabilidade de resposta para o i-ésimo fator (ou covariante)

$\alpha=$ constante

$\beta_{i}=$ coeficientes das variáveis independentes

$X_{i}=$ variáveis independentes

A técnica de regressão logística é normalmente preferida em relação a outras técnicas como a análise discriminante, segundo Hair (2005), devido a não dependência de que diversas suposições rígidas sejam atendidas e pela robustez quando estas não são satisfeitas, como a relação linear entre variáveis dependentes e independentes e a distribuição nor- mal da variável dependente e dos termos de erro.

Entretanto, segundo Garson (2008), a regressão logística ainda necessita que outros pressupostos sejam atendidos, destacando-se:

- A variável dependente deve ser dicotômica ou multinomial;

- Inclusão de todas as variáveis relevantes no modelo;

- Exclusão de todas as variáveis irrelevantes no modelo;

- Ausência de multicolinearidade;

- Ajuste adequado do modelo.

Desta forma, nas próximas seções serão apresentadas análises sobre o modelo especificado, buscando garantir que os pressupostos da técnica de regressão logística sejam atendidos.

\subsection{Especificação do modelo}

O quadro 1 apresenta as variáveis inicialmente selecionadas para serem utilizadas no modelo de regressão, sendo

Tabela 1: Composição da amostra por setores e quantidade de empresas que obtiveram patentes.

\begin{tabular}{|c|c|c|c|c|}
\hline \multirow[t]{2}{*}{ Setor } & \multicolumn{2}{|c|}{ Total } & \multicolumn{2}{|c|}{ Empresas } \\
\hline & $\mathrm{n}$ & $\%$ & $\mathrm{n}$ & $\%$ \\
\hline 12. Indústria Extrativa & 582 & 1,38 & 5 & 0,27 \\
\hline 15. Alimentos e Bebidas & 4.168 & 9,92 & 85 & 4,62 \\
\hline 17. Produtos Têxteis & 1.841 & 4,38 & 56 & 3,05 \\
\hline 18. Vestuários e Acessórios & 4.828 & 11,49 & 67 & 3,64 \\
\hline 19. Artefatos de Couro & 1.349 & 3,21 & 30 & 1,63 \\
\hline 21. Celulose e Papel & 905 & 2,15 & 23 & 1,25 \\
\hline 22. Edição e Impressão & 2.742 & 6,52 & 76 & 4,13 \\
\hline 23. Refino de Petróleo & 79 & 0,19 & 0 & 0,00 \\
\hline 24. Produtos Químicos & 1.977 & 4,70 & 229 & 12,45 \\
\hline 25. Borracha e Plásticos & 2.962 & 7,05 & 202 & 10,98 \\
\hline 26. Minerais não-Metálicos & 2.894 & 6,89 & 37 & 2,01 \\
\hline 27. Metalurgia Básica & 1.226 & 2,92 & 30 & 1,63 \\
\hline 28. Produtos Metálicos & 5.035 & 11,98 & 175 & 9,52 \\
\hline 29. Máquinas e Equipamentos & 3.045 & 7,25 & 352 & 19,14 \\
\hline 30. Equipamentos de Informática & 128 & 0,30 & 34 & 1,85 \\
\hline 31. Material Elétrico & 1.215 & 2,89 & 91 & 4,95 \\
\hline 32. Eletrônicos e comunicações & 444 & 1,06 & 84 & 4,57 \\
\hline 33. Equipamentos Médicos & 575 & 1,37 & 86 & 4,68 \\
\hline 34. Veículos Automotores & 1.171 & 2,79 & 65 & 3,53 \\
\hline 35. Outros Equip. de Transporte & 223 & 0,53 & 8 & 0,44 \\
\hline 39. Outras Indústrias & 4.635 & 11,03 & 104 & 5,66 \\
\hline Total & 42.023 & 100 & 1.839 & 100 \\
\hline
\end{tabular}


esta escolha baseada essencialmente em pesquisas anteriores sobre obtenção de patentes e inovação tecnológica, buscando incluir todas aquelas com relevância para explicar o fenômeno estudado.

Destaca-se que as variáveis incluídas no quadro 1 tomam por base os trabalhos de Watanabe, Tsuji e Brown (2004), Milson e Wilemon (2006), Hagedoorn (2002), Shefer e Frenkel (2005), Olsson e McQueen (2000), Quadros et alii (2001) e também outros elementos propostos pelos autores deste trabalho para ampliar seu potencial investigativo.

Desta forma, a construção do quadro 1 tem por objetivo satisfazer os dois primeiros pressupostos da técnica de regressão logística, identificando a variável de resposta como binária e buscando incluir todas as variáveis relevantes no modelo de regressão.

Entretanto, em face de possíveis problemas de multicolinearidade (cuja análise será feita na seção posterior), devido ao grande número de variáveis no modelo, o que provocaria uma relevante diminuição na quantidade de seu poder explicativo, foi utilizada a Análise Fatorial de Componentes Principais para as variáveis com escala de razão, buscando reduzir o modelo e qualificá-lo.

Tabela 2: Distribuição da amostra da PAEP por faixas de pessoal ocupado.

\begin{tabular}{lccc}
\hline & & $\mathrm{n}$ & $\%$ \\
\hline Total de & Até 29 Pessoas & 32.891 & 78,27 \\
empresas & 30 a 99 Pessoas & 5.904 & 14,05 \\
& 100 a 499 Pessoas & 2.546 & 6,06 \\
& 500 ou mais & 682 & 1,62 \\
\hline Empresas que & Até 29 Pessoas & 977 & 2,97 \\
obtiveram & 30 a 99 Pessoas & 406 & 6,88 \\
patentes & 100 a 499 Pessoas & 335 & 13,16 \\
& 500 ou mais & 121 & 17,74 \\
\hline
\end{tabular}

Esta decisão se justifica, pois de acordo com Dantas e De Souza (2008), o uso da análise fatorial no tratamento e configuração das variáveis explicativas as qualifica ainda mais, colocando-as em grupos de explicação que captam melhor seus efeitos sobre a variável dependente. Assim, serão utilizados fatores para compor as variáveis explicativas quantitativas do modelo.

\section{$\Delta$ s patentes brasileiras registradas no USPTO representam menos de $0,005 \%$ do total.}

O método utilizado neste trabalho foi o Varimax, destacado por Garson (2005), sendo extraídos 3 fatores, com autovalores maiores do que 1 e que explicam $64,121 \%$ da variância total, sendo este percentual considerado aceitável (HAIR et alii, 2005).

Por meio do Quadro 2 é possível perceber que as seis variáveis com escalas de razão foram reduzidas a três fatores: o fator F1 equivale ao tamanho da empresa em termos de força produtiva, o fator F2 representa os investimentos realizados pela empresa em pesquisa e desenvolvimento, já o fator F3 traduz a atratividade de profissionais qualificados que a empresa possui, unindo seu tempo de mercado ao seu salário médio.

Com o objetivo de melhorar a especificação do modelo, visando retirar variáveis que possivelmente sejam irrelevantes, foi adotado o método backwards stepwise, pelo critério do menor Likelihood Ratio. O método stepwise é baseado em um algoritmo estatístico que avalia a importância de cada variável independente e as inclui ou exclui do modelo segundo uma determinada regra. Neste estudo o parâmetro utilizado foi $10 \%$ de significância para a saída das variáveis, resultando em um modelo final composto pelo fator $\mathrm{F} 2$ e pelas variáveis X2, X3, X8, X11, X12, X14, X15 e X17.

\subsection{Análise de multicolinearidade}

Um aspecto importante a ser analisado ao utilizar a regressão logística é a possibilidade de existência de mul-

Tabela 3: Orientação exportadora e origem do capital controlador das empresas da amostra.

\begin{tabular}{|c|c|c|c|c|c|}
\hline & & \multicolumn{2}{|c|}{ Total de empresas } & \multicolumn{2}{|c|}{ Empresas que obtiveram patentes } \\
\hline & & $\mathrm{n}$ & $\%$ & $\mathrm{n}$ & $\%$ \\
\hline \multirow{2}{*}{$\begin{array}{l}\text { Orientação } \\
\text { exportadora }\end{array}$} & Exporta & 4.604 & 10,96 & 694 & 15,07 \\
\hline & Não exporta & 35.225 & 83,82 & 1.078 & 3,06 \\
\hline \multirow{2}{*}{$\begin{array}{l}\text { Origem do capital } \\
\text { controlador }\end{array}$} & Nacional & 39.012 & 92,83 & 1.557 & 3,99 \\
\hline & Internacional ou misto & 1.117 & 2,66 & 237 & 21,22 \\
\hline
\end{tabular}


ticolinearidade, que de acordo com Myers, Montgomery e Vining (2002), é um problema causado pela correlação entre variáveis independentes, o que pode levar a redução do poder explicativo do modelo de regressão.

Para a avaliação da multicolinearidade, Garson (2008) sugere a utilização do Variance Inflation Factor (VIF), que avalia o aumento da variância devido à presença de multicolinearidade e é calculado pela equação 3 :

$$
V I F=\frac{1}{1-R^{2}}
$$

De acordo com Gujarati (2000), o valor limite do VIF para estabelecer se uma variável não é colinear é 4 , sendo que se este valor for superior a 10, a variável é altamente colinear.
Assim, os valores destes indicadores encontrados para o modelo utilizado no estudo estão apresentados na Tabela 4 a seguir:

A Tabela 4 evidencia o aumento do poder explicativo em cada passo do método stepwise, mostrando que o modelo final é capaz de explicar corretamente 84,5\% (Nagelkerke $\mathrm{R}^{2}$ ) das ocorrências de obtenção de patentes na indústria do Estado de São Paulo, levando à conclusão de que o modelo foi corretamente especificado para a análise proposta neste trabalho.

\subsection{Teste de ajuste do modelo}

Uma vez definido o modelo é necessário testar a sua validade. Em regressão logística existe uma série de testes de ajuste para assegurar a validade do modelo, permitindo identificar as variáveis que não se ajustam bem, ou que têm forte influência sobre a estimação dos parâmetros.

Entre estes testes é possível destacar o de Hosmer-Lemeshow (HL), que avalia o modelo ajustado, associando os dados e suas probabilidades estimadas da mais baixa à mais alta, realizando um teste qui-quadrado para determinar a proxi-

A existência de multicolinearidade entre as variáveis pesquisadas neste trabalho foi testada pelo fator VIF, sendo que na verificação realizada nenhuma variável apresentou valor superior a 4,0, que é o valor máximo para indicar que as variáveis não são multicolineares. Portanto, nenhuma variável do modelo final especificado na seção anterior foi excluída da regressão logística.

\subsection{Análise de qualidade geral do modelo}

Para determinar o poder de explicação do modelo são utilizada, na regressão logística métricas similares às usadas na análise de regressão tradicional, em que se calcula o coeficiente de explicação ou de determinação $\left(\mathrm{R}^{2}\right)$. Na regressão logística, este coeficiente recebe a denominação de "pseudo-

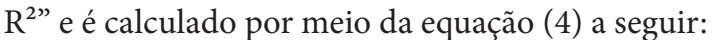

$$
R_{\log i t}^{2}=\frac{-2 L L_{\text {nulo }}-2 L L_{\text {modelo }}}{-2 L L_{\text {nulo }}}
$$

De forma similar ao pseudo- $\mathrm{R}^{2}$, podem ser utilizadas como medidas de qualidade geral do modelo o $\mathrm{R}^{2}$ de Cox e Snell e o $\mathrm{R}^{2}$ de Nagelkerque, onde, quanto maior o valor encontrado, maior será o poder explicativo do modelo.

O Cox-Snell $\mathrm{R}^{2}$ tem uma escala que começa em zero mas não alcança um em seu limite superior. O Nagelkerke $\mathrm{R}^{2}$ é similar ao Cox-Snell $\mathrm{R}^{2}$, porém tem uma escala que vai de zero a um. midade entre as frequências observadas e esperadas.

De acordo com Brito e Assaf Neto (2008), o teste HL avalia a hipótese nula de que não há diferenças significativas entre as classificações preditas e observadas, sendo que ao nível de significância de 5\% esta hipótese pode ser aceita, indicando que o modelo é capaz de produzir estimativas e classificações confiáveis.

Segundo Hosmer e Lemeshow (1989), para um adequado ajuste deve-se ter pelo menos um valor superior a 0,5 , sendo que neste trabalho o valor encontrado para o teste HL foi de 0,721, mostrando um bom ajuste para classificar a obtenção de patentes na indústria paulista.

\section{APRESENTAÇÃO E ANÁLISE DOS RESULTADOS DA REGRESSÃO LOGÍSTICA}

Os resultados encontrados com a utilização da técnica de regressão logística para a obtenção de patentes na indústria do Estado de São Paulo estão apresentados na Tabela 5, que traz as variáveis que são estatisticamente significativas (a $10 \%$ ) e os seus efeitos marginais, que representam a variação na probabilidade de obtenção de patentes quando a variável explicativa sofre um incremento ou quando assume valor igual a um (em se tratando de variáveis dummies).

A primeira variável a ser destacada nesta seção para explicar o fenômeno estudado é a orientação exportadora, 
onde os efeitos marginais mostram que empresas exportadoras possuem $1,53 \mathrm{vez}$ a probabilidade de obterem patentes do que empresas não-exportadoras. Isso provavelmente acontece devido à forte competitividade do mercado internacional, o que estimula a empresa a proteger a propriedade intelectual.

A origem do capital controlador apresenta efeitos margi- nais cujo valor mostra que empresas com origem do capital controlador internacional ou misto possuem probabilidade de obter patentes iguais a 1,48 vezes a probabilidade das empresas cujo capital controlador é de origem nacional. Este aspecto pode ser explicado pela preocupação da matriz da empresa em proteger as inovações desenvolvidas nas filiais, buscando patentear principalmente em seu país

Quadro 1: Variáveis inicialmente selecionadas para o modelo de regressão.

\begin{tabular}{|c|c|c|c|}
\hline & Variável dependente & Escala adotada & Tipo de escala \\
\hline Y1 & Obtenção de patentes no Brasil ou no Exterior & $\begin{array}{c}1=\text { Obteve } \\
0=\text { Não obteve }\end{array}$ & Nominal (dummy) \\
\hline & Variáveis Independentes & Escala adotada & Tipo de escala \\
\hline $\mathbf{X} \mathbf{1}$ & Número de funcionários & Variável contínua, $>0$ & Razão \\
\hline $\mathbf{X} 2$ & Orientação exportadora & $\begin{array}{c}1=\text { Exporta } \\
0=\text { Não exporta }\end{array}$ & Nominal (dummy) \\
\hline $\mathbf{X 3}$ & Origem do capital controlador & $\begin{array}{l}1=\text { Estrangeiro ou misto } \\
0=\text { Nacional }\end{array}$ & Nominal (dummy) \\
\hline $\mathbf{X} 4$ & Salário médio pago na empresa (R\$) & Variável contínua, $>0$ & Razão \\
\hline X5 & Idade da empresa (anos) & Variável contínua, $>=0$ & Razão \\
\hline $\mathbf{X 6}$ & Pertencer a grupo empresarial & $\begin{array}{c}1=\text { Pertence } \\
0=\text { Não pertence }\end{array}$ & Nominal (dummy) \\
\hline $\mathbf{X 7}$ & $\begin{array}{l}\text { Percentual dos recursos humanos alocados em atividades de } \\
\text { produção }\end{array}$ & Variável inteira, $>=0$ & Razão \\
\hline $\mathbf{X 8}$ & Origem da maior parte da receita da empresa & $\begin{array}{c}1=\text { Venda de bens } \\
0=\text { Venda de serviços }\end{array}$ & Nominal (dummy) \\
\hline $\mathbf{X 9}$ & $\begin{array}{c}\text { Percentual da receita investido anualmente em pesquisa e } \\
\text { desenvolvimento }\end{array}$ & Variável contínua, $>=0$ & Razão \\
\hline $\mathbf{X 1 0}$ & $\begin{array}{l}\text { Percentual dos recursos humanos alocado em atividades de } \\
\text { pesquisa e desenvolvimento }\end{array}$ & Variável contínua, $>=0$ & Razão \\
\hline $\mathbf{X} 11$ & $\begin{array}{l}\text { Laboratório ou departamento específico de pesquisa e } \\
\text { desenvolvimento }\end{array}$ & $\begin{array}{c}1=\text { Possui } \\
0=\text { Não possui }\end{array}$ & Nominal (dummy) \\
\hline $\mathbf{X 1 2}$ & Cooperação em pesquisa e desenvolvimento & $\begin{array}{c}1=\text { Coopera } \\
0=\text { Não coopera }\end{array}$ & Nominal (dummy) \\
\hline $\mathbf{X 1 3}$ & Apoio governamental na forma de subsídios ou financiamentos & $\begin{array}{c}1=\text { Recebeu } \\
0=\text { Não recebeu }\end{array}$ & Nominal (dummy) \\
\hline $\mathbf{X 1 4}$ & Fontes de informação internas para atividades de inovação & $\begin{array}{c}1=\text { Utiliza } \\
0=\text { Não utiliza }\end{array}$ & Nominal (dummy) \\
\hline X15 & $\begin{array}{l}\text { Fontes de informação ligadas ao mercado para atividades de } \\
\text { inovação }\end{array}$ & $\begin{array}{c}1=\text { Utiliza } \\
0=\text { Não utiliza }\end{array}$ & Nominal (dummy) \\
\hline X16 & Fontes de informação institucionais para atividades de inovação & $\begin{array}{c}1=\text { Utiliza } \\
0=\text { Não utiliza }\end{array}$ & Nominal (dummy) \\
\hline $\mathbf{X 1 7}$ & Outras fontes de informação para atividades de inovação & $\begin{array}{c}1=\text { Utiliza } \\
0=\text { Não utiliza }\end{array}$ & Nominal (dummy) \\
\hline
\end{tabular}


de origem, fato muito comum entre empresas com esta característica.

Outra característica significativa é a origem da maior parte do faturamento das empresas, onde aquelas que possuem a maior parte de suas receitas provinda da venda de bens, possuem probabilidade de obterem patentes 1,37 vez maior do que aquelas que prestam serviços industriais, o que pode ser explicado pelo fato de que bens podem ser patenteados e serviços não, por isso elas são naturalmente estimuladas a se proteger por meio de patentes.

Um aspecto importante nos resultados apresentados na Tabela 5 são as variáveis associadas a pesquisa e desenvolvimento, destacando-se inicialmente o fator relativo aos investimentos em P\&D (em recursos humanos ou financeiros) apresenta efeitos marginais que mostram que ao sofrer um incremento, a probabilidade de obtenção de patentes aumenta em $17 \%$. Este resultado indica que ao disponibilizar recursos para novas pesquisas, a empresa aumenta a chance de desenvolver novas tecnologias que posteriormente podem ser patenteadas.

Já os efeitos marginais da variável relativa à presença de laboratório ou departamento específico de $\mathrm{P} \& \mathrm{D}$ mostram que empresas que possuem este tipo de infraestrutura têm 1,32 vez a probabilidade de obter patentes das empresas

Quadro 2: Fatores extraídos da análise fatorial.

\begin{tabular}{cc}
\hline Fator & Definição \\
\hline F1 & $\begin{array}{c}\text { Tamanho da empresa (Número de funcionários } \\
\text { e porcentagem de funcionários alocados em } \\
\end{array}$ \\
atividades de produção). \\
F2 & Investimentos em P\&D (Investimentos financeiros \\
& e de recursos humanos em P\&D). \\
& Atratividade profissional (Idade da empresa \\
& e Salário médio pago na empresa).
\end{tabular}

Tabela 4: Poder explicativo do modelo de regressão logística.

\begin{tabular}{ccc}
\hline Step & Cox \& Snell $\mathbf{R}^{2}$ & Nagelkerque $\mathbf{R}^{\mathbf{2}}$ \\
\hline 1 & 0,422 & 0,561 \\
2 & 0,561 & 0,734 \\
3 & 0,604 & 0,801 \\
4 & 0,614 & 0,812 \\
5 & 0,631 & 0,845 \\
\hline
\end{tabular}

que não possuem. Isto se explica por se tratar de ambientes específicos para a geração de conhecimentos e tecnologias aplicáveis à indústria e, portanto, altamente patenteáveis.

Também se deve destacar a cooperação em P\&D, cujos resultados apontam que a probabilidade obterem patentes das empresas que realizam cooperação é igual a $1,21 \mathrm{vez}$ a probabilidade das que não realizam este tipo de cooperação. Este resultado pode ser explicado pela maior capacidade de investimento e complementaridade de recursos quando a empresa realiza suas atividades de P\&D de forma cooperada.

Em se tratando de fontes de informação para atividades de inovação, a variável, relativa às fontes internas, mostra que empresas que atribuíram importância a este tipo de fontes possuem probabilidade de obterem patentes igual a $1,29 \mathrm{vez}$ a probabilidade de empresas que não atribuíram, o que pode ser explicado pelo fato de que o conhecimento gerado dentro da própria empresa (no departamento de $\mathrm{P} \& \mathrm{D}$ ou em outras áreas) tem um caráter diretamente aplicável ao seu próprio negócio, gerando inovações em produtos e processos e estimulando a sua proteção por parte das empresas por meio de patentes.

Em relação às fontes ligadas ao mercado, surpreendentemente os resultados mostram que empresas que atribuíram importância a estes tipos de fontes têm probabilidade de obter patentes igual a apenas $0,72 \mathrm{vez}$ a probabilidade das empresas que não atribuíram, tendo, portanto, uma influência negativa, o que se explica pelo fato de que embora o conhecimento provindo do mercado seja útil, muitas vezes já é de propriedade de outras empresas, não podendo portanto ser patenteado.

Tabela 5: Resultados do modelo de regressão logística.

\begin{tabular}{ccc}
\hline & Variáveis Explicativas & $\begin{array}{c}\text { Efeitos } \\
\text { marginais }\end{array}$ \\
\hline X2 & Orientação exportadora & $\mathbf{1 , 5 3}$ \\
$\mathbf{X 3}$ & Origem do capital controlador & $\mathbf{1 , 4 8}$ \\
$\mathbf{X 8}$ & Origem da maior parte da receita & $\mathbf{1 , 3 7}$ \\
& (bens ou serviços) & \\
$\mathbf{F 2}$ & Fator F2 (investimentos em P\&D) & $\mathbf{0 , 1 7}$ \\
$\mathbf{X 1 1}$ & Laboratório de P\&D & $\mathbf{1 , 3 2}$ \\
$\mathbf{X 1 2}$ & Cooperação em P\&D & $\mathbf{1 , 2 1}$ \\
$\mathbf{X 1 4}$ & Fontes internas & $\mathbf{1 , 1 9}$ \\
$\mathbf{X 1 5}$ & Fontes ligadas ao mercado & $\mathbf{0 , 7 2}$ \\
$\mathbf{X 1 7}$ & Outras fontes & $\mathbf{2 , 1 9}$ \\
\hline
\end{tabular}


A última variável estatisticamente significativa é a atribuição de importância às chamadas outras fontes (feiras, eventos ou aquisição de licenças), cujos resultados mostram que as empresas industriais do estado que atribuíram importância para este tipo de fontes possuem probabilidade de obterem patentes igual a 2,19 vezes a probabilidade de empresas que não atribuíram. Este resultado indicar, que ao ter contato com este tipo de eventos, onde os produtos e processos já estão protegidos por meio de patentes, ou mesmo ao adquirir novas licenças e patentes, as empresas ficam estimuladas a proteger seu próprio patrimônio intelectual, o que explica a maior probabilidade de se obter patentes.

\section{CONCLUSÕES}

Esta pesquisa teve por objetivo analisar as variáveis de influência na obtenção de patentes na indústria do Estado de São Paulo. Para atingir este objetivo foi construído um modelo de regressão logística que relacionou diversas variáveis que podem influenciar a probabilidade de obtenção de patentes nestas empresas.

Os resultados mostram que o modelo utilizado possui um poder explicativo expressivo quando aplicado para explicar o fenômeno estudado, sendo capaz de explicar cerca de $84,5 \%$ dos casos de obtenção de patentes, além disso o modelo foi testado quanto à multicolinearidade (VIF < 4,0 para todas as variáveis) e quanto ao ajuste (teste $\mathrm{HL}$ $=0,721)$, o que permite a conclusão de que o modelo foi corretamente especificado.

Os resultados encontrados permitem concluir que as variáveis significativas para explicar o fenômeno estudado são: a orientação exportadora, a origem do capital controlador, a origem da maior parte da receita, os investimentos em $\mathrm{P} \& \mathrm{D}$, a presença de laboratório ou departamento específico de $P \& D$, a cooperação em P\&D, atribuição de importância para fontes internas, ligadas ao mercado e outras fontes de informação.

Estes resultados confirmam estudos empíricos presentes na literatura, como os de Salomon e Shaver (2005) (orien- tação exportadora), De Negri e Turchi (2007) (origem do capital controlador), Santarelli e Sterlachini (presença de laboratório ou departamento específico de P\&D), Dowling e Helm (Cooperação em P\&D) e Quadros et alii (2001) (fontes de informação para atividades inovativas).

\section{E ntender este perfil pode auxiliar na criação subsídios para a implementação de políticas públicas.}

Entre os resultados é possível destacar aqueles relacionados a pesquisa e desenvolvimento (investimentos, infra-estrutura específica e cooperação), mostrando a importância deste elemento no desenvolvimento de novas tecnologias comerciais que possam ser patenteadas pelas empresas.

É preciso ressaltar que estes resultados são relativos à indústria paulista de forma agregada e que setorialmente (considerando os vinte e um setores com significância estatística na PAEP) existem diferenças quanto a quais variáveis impactam a probabilidade de obtenção de patentes e qual a contribuição de cada variável para o aumento ou a redução desta probabilidade, como pode ser verificado em Pacagnella Júnior (2006).

De modo geral os resultados encontrados por este trabalho apresentam como contribuição a junção de diversos aspectos relevantes em um mesmo modelo teórico-empírico que permitiu traçar um perfil da empresa com maior chance de obter patentes e qual a influência das variáveis que compõem este perfil.

Entender este perfil pode auxiliar na criação de estratégias tecnológicas ou ainda fornecer subsídios para a implementação de políticas públicas que fomentem a inovação tecnológica por parte das empresas.

Desta forma, espera-se que este trabalho possa contribuir para a compreensão do fenômeno estudado e de outros relacionados. Além disso, deixa-se como sugestão a realização de estudos semelhantes, baseados em surveys em outros estados ou nos setores industriais mais importantes economicamente ou de maior relevância tecnológica, de modo a verificar a validade e relevância do modelo formulado ou ainda encontrar outras variáveis não discutidas neste trabalho. 


\section{REFERÊNCIAS}

ABRAHAM, B. P.; MOITRA, S. D. Innovation assessment through patent analysis. Technovation, v. 21, n. 4 , p. $245-252,2001$.

ALBUQUERQUE, E. M.; SIMÕES, R.; BAESSA, A.; CAMPOLINA, B.; SILVA, L. A Distribuição espacial da produção científica e tecnológica brasileira: uma descrição de estatísticas de produção local de patentes e artigos científicos. Revista Brasileira de Inovação, v. 1, n. 2, p. 225-251, 2002.

BRITO, G.; ASSAF NETO. A. Modelo de Classificação de Risco de Crédito nas Empresas. Revista Contabilidade \& Financas, v. 19, n. 2, p. 18-29, 2008.

BURKE, P. F.; REITZIG, M. Measuring patent assessment quality: Analyzing the degree and kind of (in)consistency in patent offices decision making, Research Policy, v. 36, n. 9 p. 1404-1330, 2007.

CHOY, C. S.; YEW, W. K.; LIN, B. Criteria for measuring $\mathrm{KM}$ performance outcomes in organizations, Industrial Management \& Data Systems, v. 106, n. 7, p. 917-36, 2006.

COOMBS, J. E.; BIERLY, P. E. Measuring technological capability and performance. $R \& D$ Management, v. 36, n. 4, p. 421-438, 2006.

DANE, F. C. Research Methods. Brooks/Cole Publish Company, Pacific Groove, California, United States, 1990.

DANTAS, R. F.; DESOUZA, S. A. Modelo de risco e decisão de crédito baseado em estrutura de capital com informação assimétrica. Pesquisa Operacional, v. 28, n. 2, p. 263-284, 2008.

DE NEGRI, J. A.; TURCHI, L. M. Technological innovation in Brazilian and Argentine firms. Brasília: IPEA, 2007.

DOWLING, M.; HELM, R. Product development success through cooperation: A study of entrepreneurial firms, Technovation, v. 26, n. 1, p. 483-488, 2006.

ENCAOUA, D.; GUELLEC, D.; MARTíNEZ, C. Patent systems for encouraging innovation: Lessons from economic analysis. Research Policy, v. 35, n. 9, p. 1423-1440, 2006.
FAPESP. Indicadores de C\&T e Inovação em São Paulo, São Paulo: Editora FAPESP, 2004.

GARSON, G. D. Quantitative Research in Public Administration. NC State University, 2005. Disponível em: http://www2.chass.ncsu. edu/garson/pa765/factor.htm. Acesso em: 10 nov. 2008.

GIL, A. C. Como elaborar projetos de pesquisa. São Paulo: Atlas, 2002.

GUJARATI, D. N. Econometria Básica. 3. ed. São Paulo: Makron Books, 2000.

HAGEDOORN, J. Measuring innovative performance: is there an advantage in using multiple indicators? Research Policy, v. 32, n. 1, p. 1365-1379, 2002.

HAIR, J. F. J.; ANDERSON, R. E.; TATHAM, R L.; BLACK, W. C. Multivariate Data Analysis, Ed. Englewood Cliffs: NJ: Prentice-Hall Inc., 2005.

HOSMER, D. W. LEMESHOW, S. Applied Logistic Regression. Wiley, New York, 1989.

HOU, J. L.; LIN, H. Y. A multiple regression model for patent appraisal. Industrial Management \& Data Systems, v. 106, n. 9, p. 1304-1332, 2006.

HUMPHREYS, L. F. L. Análise da proteção das patentes segundo a lei brasileira. Revista Brasileira de Direito Internacional, v. 4, n. 4, p. 158-171, 2006.

JOHNSON, R. A.; WICHERN, D. W. Applied multivariate statistical analysis. New Jersey: Prentice-Hall, 1998.

MILSON, R.; WILEMON, D. The impact of organizational integration and product development proficiency on market sucess. Industrial Marketing Management, v. 31, n. 1, p. 1-23. 2002.

MYERS, R. H.; MONTGOMERY, D. C.; VINING, G. G. Generalized Linear Models with Applications in Engineering and Sciences. New York: Wiley, 2002.

OCDE. Compendium of Patent Statistics, Disponível em http://www.oecd.org. Acesso em 14 dez 2007.
OCDE, Compendium of Patent Statistics, Ed. OCDE: Paris, 2007

OLSSON, H.; MCQUEEN, D. H. Factors influencing patenting in small computer software producing companies. Technovation, v. $20, \mathrm{n}$. 10, p. 563-576, 2000.

PACAGNELLA JÚNIOR, A. C. A inovação tecnológica nas indústrias do Estado de São Paulo: uma análise dos indicadores da PAEP. Dissertação de mestrado, 243 p. Faculdade de Economia, Administração e Contabilidade de Ribeirão Preto, Universidade de São Paulo, 2006.

QUADROS, R.; FURTADO, A.; BERNARDES, R.; FRANCO, E. Technological innovation in Brazilian industry: an assessment based on the São Paulo innovation survey, Technological forecasting and social change, v. 67, n. 2 , p. 203-219, 2001.

REITZIG, M. What determines a patent value? Insights from semiconductor industry. Research Policy, v. 32, n. 1 , p. 13-26, 2001.

RICHARDSON, R. J. e colaboradores. Pesquisa social: métodos e técnicas. São Paulo: Atlas, 1999.

SALOMON, M. R.; SHAVER, M. J. Learning by exporting: new insights from examining firm innovation, Journal of Economics \& Management Strategy, v. 14, n. 2, p. 431-460, 2005

SANTARELLI, E.; STERLACCHINI, A. Innovation, formal vs. informal R\&D, and firm size: Some evidence from Italian manufacturing firms. Small Business Economics, v. 2, n. 3, p. 223-228, 2004.

SHEFER, D.; FRENKEL, A. R\&D, firm size and innovation: an empirical analysis, Technovation, v. 25 n. 1, p. 25-32, 2005 .

WATANABE, C.; TSUJI, Y.; BROWN, G. C. Patent statistics: deciphering a "real" versus a "pseudo" proxy of innovation, Technovation, v. 21, n. 1, p. 783-790, 2001. 


\section{SOBRE OS AUTORES}

\section{Antônio Carlos Pacagnella Júnior}

Departamento de Engenharia de Produção / UFSCar

End.: Lindoro Vicente Santana, 190, Sumarezinho - Ribeirão Preto/SP

Tel.: (16) 3630-2163 Fax: (16) 3630-2163

E-mail: acpjr@usp.br

\section{Geciane Silveira Porto}

Departamento de Administração - FEARP/USP

End.: Av. dos Bandeirantes, 3900, Monte Alegre - Ribeirão Preto/SP

Tel.: (16) 3602-3914 Fax: (16) 3602-3922

E-mail: geciane@usp.br

\section{Sérgio Kannebley Júnior}

Departamento de Economia - FEARP/USP

End.: Av. dos Bandeirantes, 3900, Monte Alegre - Ribeirão Preto/SP

Tel.: (16) 3602-3887 Fax: (16) 3602-3922

E-mail: skj@usp.br

\section{Sérgio Luís da Silva}

Departamento de Engenharia de Produção / UFSCar

End.: Rua 28 de Setembro, 2360, ap. 41T, Centro, São Carlos/SP

Tel.: (16) 3352-8239 Fax: (16) 3351-8240

E-mail: sergiol@power.ufscar.br

\section{Carlos Alberto Grespan Bonacim}

Departamento de Contabilidade / FEA-USP

End.: Rua Romeu Engrácia de Faria, 210, ap. 15-A

Tel.: (16) 8118-9360

E-mail: carlosbonacim@yahoo.com.br 